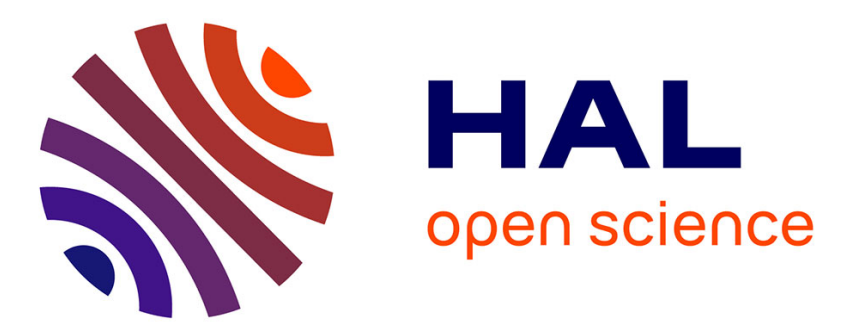

\title{
Assessment of muscle hardness changes induced by a submaximal fatiguing isometric contraction
}

\author{
Antoine Nordez, Arnaud Guével, Pascal Casari, Stefan Catheline, Christophe
}

Cornu

\section{- To cite this version:}

Antoine Nordez, Arnaud Guével, Pascal Casari, Stefan Catheline, Christophe Cornu. Assessment of muscle hardness changes induced by a submaximal fatiguing isometric contraction. Journal of Electromyography and Kinesiology, 2009, 19 (3), pp.484-91. 10.1016/j.jelekin.2007.11.005 . hal01005278

\section{HAL Id: hal-01005278 \\ https://hal.science/hal-01005278}

Submitted on 20 Feb 2017

HAL is a multi-disciplinary open access archive for the deposit and dissemination of scientific research documents, whether they are published or not. The documents may come from teaching and research institutions in France or abroad, or from public or private research centers.
L'archive ouverte pluridisciplinaire HAL, est destinée au dépôt et à la diffusion de documents scientifiques de niveau recherche, publiés ou non, émanant des établissements d'enseignement et de recherche français ou étrangers, des laboratoires publics ou privés.

\section{(c)(1)}

Distributed under a Creative Commons Attribution| 4.0 International License 


\title{
Assessment of muscle hardness changes induced by a submaximal fatiguing isometric contraction
}

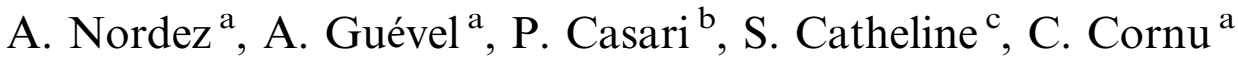 \\ a Université de Nantes, Nantes Atlantique Universités, Laboratoire "Motricité, Interactions, Performance", JE 2438, UFR STAPS, \\ 25 bis Bd Guy Mollet BP 72206, Nantes F-44000, France \\ ${ }^{\mathrm{b}}$ Université de Nantes, Nantes Atlantique Universités, CNRS, Institut de recherche en Génie Civil et Mécanique, UMR 6183, \\ UFR des Sciences et Techniques, 2 rue de la Houssinière BP 92208, Nantes F-44000 France \\ ${ }^{\circ}$ Laboratoire Ondes et Acoustique, UMR CNRS 7587, ESPCI, Université Paris VII, France
}

Transient elastography consists of measuring the transverse local shear elastic modulus defined as local muscle hardness (LMH). It has previously been shown that LMH is correlated to muscle activity level during non-fatiguing contractions. The aim of this study was to describe how LMH and muscle activity level change during a submaximal fatiguing constant-torque protocol. Changes in gastrocnemius medialis LMH and in surface electromyographic activities (sEMG) of plantar flexors induced by a submaximal isometric plantar flexion ( $40 \%$ of the maximal isometric torque) until exhaustion were quantified. During the contraction, sEMG of each muscle increased $(P<0.001)$ whereas LMH remained constant $(P>0.05)$. Active LMH assessed during the contraction did not parallel muscle activity level changes during this type of submaximal fatigue protocol. Interestingly, LMH at rest assessed in passive conditions was higher prior to the fatiguing effort $(P<0.05)$, rather than that assessed immediately after. Muscle and tendon viscous behaviors could imply a creep phenomenon during a prolonged isometric contraction, and our results in LMH at rest could indicate that this phenomenon induces changes in muscle intrinsic mechanical properties. Further studies are needed to examine whether it could have an influence on muscle activity levels during the contraction.

Keywords: Muscle hardness; Stiffness; Creep; Fatigue; Surface electromyography

\section{Introduction}

Muscle fatigue induces an increase in muscle activity required to sustain a contraction at a constant force level. This phenomenon could be interpreted as a decrease in muscle efficiency, and has been intensively studied in terms of changes in neural control mechanisms (De Luca, 1984). Surface electromyography (sEMG) is a useful non-invasive means of studying the neuromuscular system during fatigue (e.g. De Luca, 1997). Typically, during a submaximal isometric contraction sustained until exhaustion, an increase in sEMG activity is usually an indication of an enhancement of the central drive as a result of the recruitment of additional motor units and a modulation of the discharge rate to compensate for the development of peripheral fatigue (De Luca, 1984; Fuglevand et al., 1993). On the other hand, a compression of the sEMG power spectrum towards lower frequencies is suggested to be mainly related to a decrease in muscle fiber conduction velocity due to an accumulation of metabolites reducing the intracellular pH (Brody et al., 1991; Lindstrom et al., 1977).

Viscoelasticity, which is observed in both active and passive muscle-tendon behavior (Fung, 1993), has been shown to contribute significantly to muscle efficiency (e.g. Hof, 
2003; Wilson et al., 1994). Additionally, musculo-articular stiffness determines resistance to external perturbations and has important implications for neuromuscular control (Hof, 2003; Hunter and Kearney, 1983). As a consequence, a potential alteration in musculo-articular mechanical behavior induced by a prolonged fatiguing contraction would influence changes in neural control mechanisms and sEMG recordings. Nevertheless, the few studies that have investigated changes in musculo-articular mechanical behavior induced by fatigue produced controversial results. Two studies have shown that both series elastic stiffness of quadriceps muscles (Vigreux et al., 1980) and elbow musculo-articular stiffness (Zhang and Rymer, 2001) are decreased after an isometric fatiguing contraction using the quick release method and the application of dynamical perturbations, respectively. In contrast, Hunter and Kearney (1983), using dynamical perturbations, have shown that ankle musculo-articular stiffness was not significantly changed by a submaximal isometric prolonged plantar flexion.

The methods used in these older studies cited above are still very useful for the understanding of in vivo muscle function, but they only provide global measurement of a muscletendon unit, or a musculo-articular complex mechanical behavior, specifically in terms of stiffness. On the contrary, the use of ultrasonography has enabled the mechanical characterization of localized structures such as tendon (Kubo et al., 2001; Mademli et al., 2006; Maganaris et al., 2006). However, ultrasonography is limited to the characterization of superficial structures and remains difficult to use to assess muscle elastic properties. New methodologies are needed to follow changes in muscle stiffness during a prolonged contraction, and are discussed later, herein.

The non-invasive assessment of localized muscle mechanical properties would enable estimation of individual muscle contributions to global joint stiffness, and remains a topic of continued interest to researchers. While all of the above studies concern the assessment of the stiffness along the longitudinal axis of the muscle, another approach called elastography has been developed to characterize a transverse muscle stiffness that has been termed 'hardness'. More precisely, elastography consists of measuring the velocity of transverse shear wave propagation, which is related to the local muscle hardness (LMH) (see Eq. (1)). This technique would allow for assessment of hardness of both superficial and deep structures. A few studies using elastographic methods have succeeded in assessing in vivo human LMH (Gennisson et al., 2005; Heers et al., 2003; Jenkyn et al., 2003; Levinson et al., 1995). Jenkyn et al. (2003) have pointed out that LMH could be correlated with muscle tension, whereas two other studies have shown that LMH was correlated with muscle activity level during non-fatiguing isometric contractions (Gennisson et al., 2005; Heers et al., 2003). Results obtained in these studies were collected using different elastographic methods. Since sonoelastography (Levinson et al., 1995) and magnetic resonance elastography (Heers et al., 2003; Jenkyn et al., 2003) require long acquisition time and imply static measurements, these methods cannot be used to study acute LMH changes induced by a fatiguing protocol. In contrast, transient elastography (Gennisson et al., 2005; Sandrin et al., 2002) provides instantaneous measurement and can be used to follow LMH changes during a contraction.

Therefore, in order to have a better understanding of muscle mechanical behavior during a fatiguing protocol, the aim of the present study was to quantify LMH changes using transient elastography induced by a submaximal plantar flexion until exhaustion. The present study would then enable to determine whether muscle LMH changes parallels muscle activity during such a fatiguing protocol. It would also have interesting implications relative to possible muscle input data for a direct dynamic model.

\section{Materials and methods}

\subsection{Subjects}

Eight healthy male subjects $(26.3 \pm 4.9$ years, $181.8 \pm$ $7.7 \mathrm{~cm}, 77.3 \pm 10.9 \mathrm{~kg}$ ) volunteered to participate for the present study and signed an informed consent form. This study was conducted according to the Helsinki Statement (1964). Subjects practiced recreational sports, but did not participate in any strength or flexibility training at the time of the study. No subjects had sustained a recent injury that might affect the findings.

\subsection{Materials}

The Biodex system 3 research $^{\circledR}$ (Biodex medical, Shirley, NY, USA) isokinetic dynamometer was used to measure torque production of ankle plantar flexors.

The shear elasticity probe (Fig. 1) used to assess LMH in the present study has previously been described (Gennisson et al., 2005; Sandrin et al., 2002). Briefly, an ultrasonic transducer (diameter: $7 \mathrm{~mm}$; focal depth: $35 \mathrm{~mm}$, Vermon, Tours, France) was set up on a vibrator (Brüel \& Kjær, type 4810, DK-2850 Nærum, Denmark). The vibrator impacted gastrocnemius medialis muscle with a low-frequency sinusoidal pulse $(100-150 \mathrm{~Hz}$, amplitude of $1 \mathrm{~mm})$ controlled by a conventional function generator. The pulse induces the propagation of a compression wave and a shear wave. Transient elastography consists of measuring shear wave velocity using ultrasound propagation, and the LMH is related to this velocity (Gennisson et al., 2005; Sandrin et al., 2002) as shown in the following equation:

$\mathrm{LMH}=\rho V_{\mathrm{S}}^{2}$

where $\rho$ is the muscle mass density $\left(\rho \approx 1000 \mathrm{~kg} \mathrm{~m}^{-3}\right.$, (Gennisson et al., 2005)) and $V_{\mathrm{S}}$ is the shear wave velocity.

The transducer works as a pulse-echo system with a $2 \mathrm{kHz}$ recurrence frequency in order to assess displacements induced by the low frequency impulse. Ultrasonic signals $(5 \mathrm{MHz}$ central frequency) were sampled at 


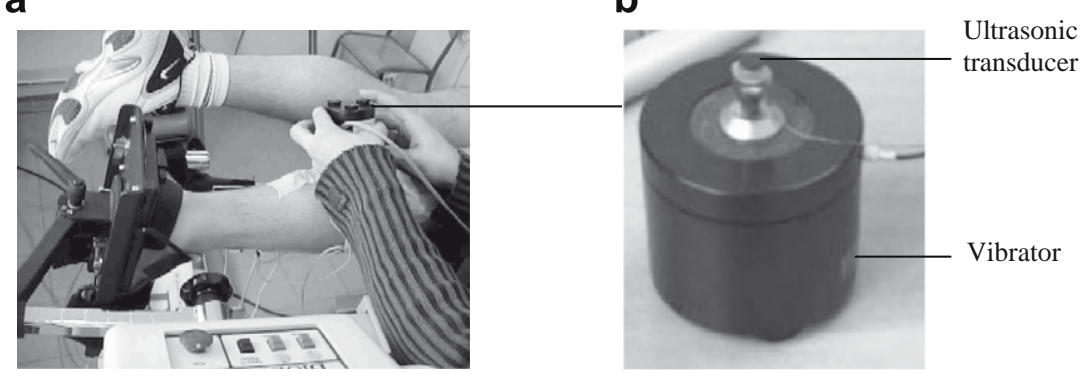

Fig. 1. Measurement techniques: (a) position of the subject on the dynamometer: subjects were lying prone, their right knee was fully extended and isometric ankle plantar flexions were performed with the foot perpendicular to the leg and (b) shear elasticity probe (Sandrin et al., 2002): it was composed of a vibrator to induce the propagation of the shear wave and of an ultrasonic transducer to measure shear wave velocity.

$50 \mathrm{MHz}$ and stored on a computer hard drive using a 9-bit digitizer with $2 \mathrm{MB}$ memory for further analysis. LMH measurements were performed in less than $50 \mathrm{~ms}$.

Bipolar surface electromyographic (sEMG) signals were recorded from surface electrodes $(\mathrm{Ag} / \mathrm{AgCl}, 4 \mathrm{~mm}$ recording diameter, In Vivo Metric, Healdsburg, CA, USA) placed on gastrocnemius lateralis (GL), gastrocnemius medialis (GM) and soleus (SO) muscles with an $11 \mathrm{~mm}$ inter-electrode distance. According to the surface electromyography for the non-invasive assessment of muscles (SENIAM) recommendations (Hermens et al., 2000), surface electrodes were placed with respect to the assumed direction of the fibers. Three reference electrodes were placed over lateral and medial malleolus. The inter-electrode resistance was reduced to less than $55 \mathrm{k} \Omega$ (Hewson et al., 2003) after standard skin preparation procedures: shaving, slight abrasion and cleaning with an alcohol-acetone solution (Maïsetti et al., 2002a). sEMG signals were pre-amplified (gain $=600$ ).

The isometric torque, the low-frequency sinusoidal pulse sent with the conventional function generator (for synchronization purposes, between LMH and sEMG measurements) and sEMG signals were sampled at 256, 512 and $1024 \mathrm{~Hz}$ respectively with a 12-bit Analogic/Digital converter (Myodata ${ }^{\circledR}$, Electronique du Mazet, France, input impedance $=10 \mathrm{G} \Omega$, common mode-rejection ratio at $50 \mathrm{~Hz}=100 \mathrm{~dB}$ ). Data were finally stored in a flash memory card and transferred to a computer hard drive for further analysis.

\subsection{Experimental setup}

Subjects were lying prone on the dynamometer and their right leg was fully extended (Fig. 1a). The input axis of the dynamometer was aligned with the supposed axis of rotation passing through the mid-point of the line connecting both malleoli of the right ankle. All tests were performed with the right foot perpendicular to the leg, and the shear elasticity probe was placed at the middle of the GM muscle belly, $1-2 \mathrm{~cm}$ lateral to surface electrodes (Fig. 1a). The experimental protocol is presented in Fig. 2. A 5 min warm-up, consisting of gradually and increased isometric plantar flexions, was performed first. After a $5 \mathrm{~min}$ rest period, the maximal voluntary isometric torque (MVIT) was assessed as the highest torque value produced from three successive plantar flexion efforts of $5 \mathrm{~s}$ separated by a $45 \mathrm{~s}$ rest period. Next, after a $10 \mathrm{~min}$ rest period, subjects were required to maintain an isometric submaximal plantar flexion at $40 \%$ of the MVIT, until exhaustion. During the test, the target torque was displayed on a computer screen. The trial was stopped when torque output dropped below $95 \%$ of the target torque. The duration of the test (Tlim) was then assessed. Active LMH assessments were performed at every $2.5 \mathrm{~s}$ during the fatiguing protocol. Before and immediately after this task, subjects were asked to relax completely, and $10 \mathrm{LMH}$ at-rest measurements were performed in $25 \mathrm{~s}$ during each of those two rest periods.

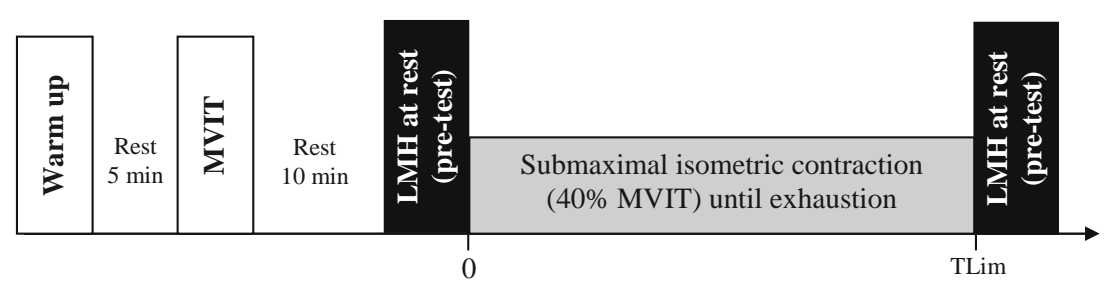

Fig. 2. Experimental protocol. After a standardized warm-up, maximal voluntary isometric torque (MVIT) in plantar flexion was assessed. Next, after a 10 min rest period, subjects were required to maintain an isometric submaximal contraction at $40 \%$ MVIT of plantar flexor until exhaustion (Tlim). Surface electromyographic and local muscle hardness (LMH) measurements were performed synchronously. Ten LMH at rest measurements were performed before (pre-test) and after (post-test) the submaximal contraction. 


\subsection{Data processing}

All of the data were processed using standardized softwere computed with Matlab ${ }^{\circledR}$ (The Mathworks, Natick, USA). Transient elastography data-processing steps were described by Gennisson et al. (2005). A cross correlation algorithm was used to assess strain field induced by the low frequency pulse. From such strain field, shear wave velocity and LMH (Eq. (1)) were calculated across muscle depth using an inversion algorithm. Torque and sEMG signals were filtered using a low-pass $(10 \mathrm{~Hz})$ and a band-pass (6-400 Hz) second-order Butterworth filters, respectively. Filters were then applied in a reverse direction of time to prevent the shift of the filter. The time corresponding to LMH measurements was determined using the low-frequency sinusoidal pulse sent with the conventional function generator and stored by the Analogic/Digital Converter (Myodata $^{\circledR}$, Electronique du Mazet, France). Analysis of sEMG signals was then performed for the GL, GM and SO muscles in the time and frequency domains using $200 \mathrm{~ms}$ windows centered over each LMH measurements. The root mean square (RMS) and the fast Fourier transform (FFT, 1024 points) of sEMG signals were calculated for each window. The mean power frequency (MPF) of the power spectrum provided by the FFT (De Luca, 1984) and the relative energy in the frequency band $6-60 \mathrm{~Hz}$ (FB1) (Maïsetti et al., 2002a) were then evaluated. The frequency band $6-60 \mathrm{~Hz}$ was chosen after a pilot analysis, which has shown that this band was the most altered by muscle fatigue. RMS values assessed during the fatiguing protocol were normalized with RMS values calculated using a $200 \mathrm{~ms}$ window placed around the peak torque obtained during the best maximal isometric contractions (RMSmax). In addition, MPF values were expressed in percentage of initial values (Maïsetti et al., 2002b). Finally, active LMH, normalized RMS and MPF values, FB1 and torque were averaged every $5 \%$ of Tlim.

\subsection{Statistics}

Ten separate one-way $(1 \times 20)$ ANOVAs with repeated measures of time were performed to assess changes in active LMH, normalized RMS, MPF and FB1 during the fatiguing protocol. LSD post hoc analyses were used when appropriate. A paired $t$-test was also performed to compare $\mathrm{LMH}$ at rest, averaged for the ten measurements performed before and immediately after this fatiguing protocol. The critical level of significance in the present study was set at $P<0.05$. Data are expressed as means \pm standard deviation.

\section{Results}

The mean duration of the fatiguing test (Tlim) was $246 \pm 83$ s (range: $131-376$ s).

Averaged values (over subjects) of the normalized RMS at the beginning of the fatiguing contraction were
$40.7 \pm 31.5 \%, 26.1 \pm 12.3 \%$ and $43.1 \pm 10.2 \%$ for $\mathrm{GM}$, GL and SO muscles, respectively. The RMS of the three muscles increased with time during the fatiguing protocol $(P<0.001$, Fig. 3a). The RMS values were significantly higher than initial values at $27.5 \%, 12.5 \%$ and $72.5 \%$ of the Tlim for GM, GL and SO muscles, respectively. At
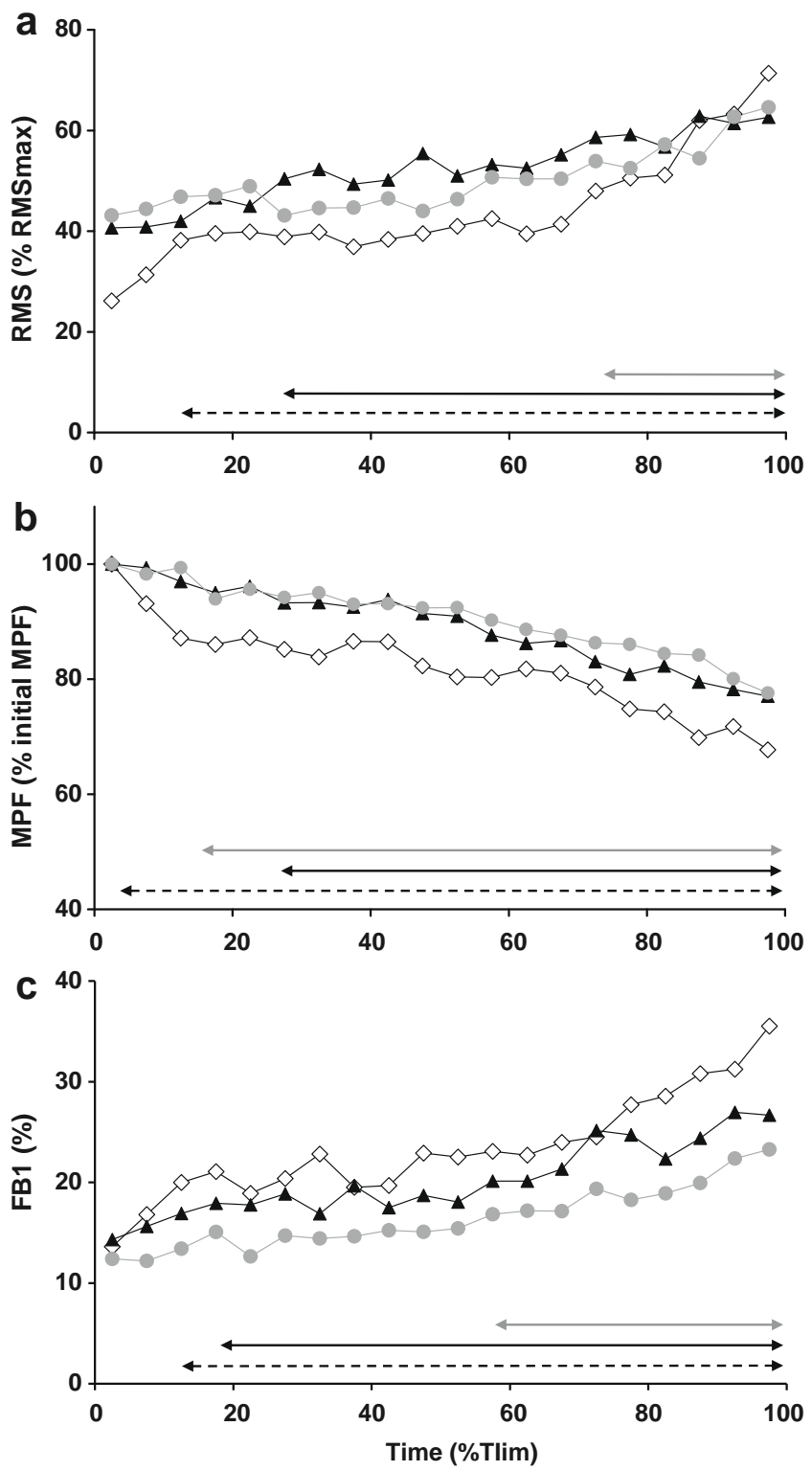

Fig. 3. (a) Changes in averaged (over subjects) root mean square of electromyographic signals (RMS) expressed in percentage of maximal activities during maximal voluntary contractions (RMSmax) as function of percentage of exhaustion time ( $\%$ Tlim) during the submaximal isometric contraction, (b) Changes in the averaged (over subjects) power frequency of electromyographic signals (MPF) expressed in percentage of the initial MPF as function of time ( $\%$ Tlim) and (c) changes in the averaged (over subjects) relative energy in the frequency band $6-60 \mathrm{~Hz}$ of electromyographic signals (FB1) as function of time. Gastrocnemius lateralis $(\diamond)$, gastrocnemius medialis $(\boldsymbol{\Delta})$ and soleus $(\ominus)$. Horizontal arrows: significant difference $(P<0.05)$ from initial values for gastrocnemius lateralis (dashed black line), gastrocnemius medialis (solid black line) and soleus (solid grey line). 
the end of the protocol, RMS values were lower than RMSmax $(62.6 \pm 24.2 \%, 71.4 \pm 35.3 \%, 64.6 \pm 18.6 \%$ for GM, GL and SO respectively). Averaged (over subjects) initial values of the MPF and FB1 were $145.2 \pm 20.5 \mathrm{~Hz}$, $142.3 \pm 18.7 \mathrm{~Hz}, 149.0 \pm 18.3 \mathrm{~Hz}$ and $16.9 \pm 7.2 \%, 18.9 \pm$ $4.0 \%, 13.4 \pm 6.9 \%$ for GM, GL, SO muscles, respectively. For the three muscles, the MPF decreased for the three muscles $(P<0.001)$ and final values were $77.1 \pm 7.9 \%$ for GM muscle, $67.7 \pm 14.9 \%$ for GL muscle and $77.6 \pm$ $6.9 \%$ for SO muscle of initial MPF values (Fig. 3b). In contrast, the FB1 increased with time $(P<0.001$; GM: $+12.4 \pm 8.9 \%$, GL: $+21.9 \pm 13.6 \%$, GM: $+10.9 \pm 8.9 \%$; Fig. 3c).

The mean active LMH was not significantly changed during the submaximal isometric contraction until exhaustion $(P>0.05$, Fig. 4).

Averaged (over time and subjects) LMH during the fatiguing contraction $(7.1 \pm 2.2 \mathrm{kPa})$ was higher than LMH at rest $(3.3 \pm 0.9 \mathrm{kPa})$. The ratio between active $\mathrm{LMH}$ and $\mathrm{LMH}$ at rest was $2.1 \pm 0.6$. LMH at rest was significantly lower after than before the fatiguing protocol

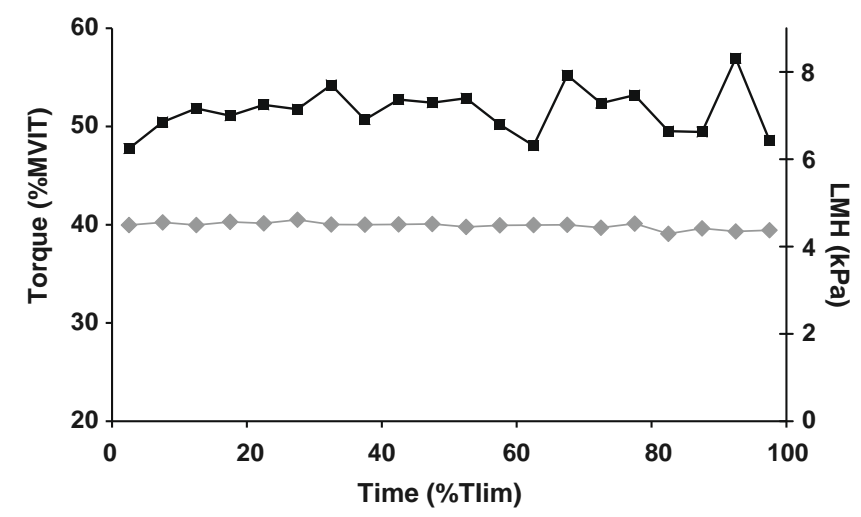

Fig. 4. Changes in averaged (over subjects) produced torque expressed in percentage of maximal voluntary isometric torque (MVIT, $\diamond$ ), averaged (over subjects) local muscle hardness $(\mathrm{LMH}, \mathbf{\square})$ as functions of percentage of exhaustion time (\% Tlim) during the submaximal isometric contraction.

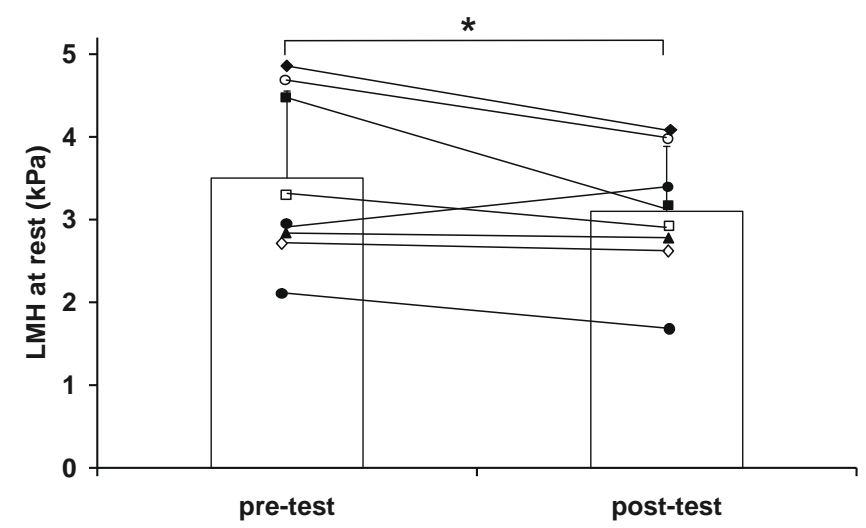

Fig. 5. Changes in local muscle hardness assessed in passive condition (LMH at rest) before (pre-test) and after (post-test) the fatiguing isometric contraction. *: $P<0.05$.
$(3.5 \pm 1.0 \mathrm{kPa}$ pre-test vs. $3.1 \pm 0.8 \mathrm{kPa}$ post-test, $P<0.05)$, as shown in Fig. 5.

\section{Discussion}

Transient elastography enables assessment of a local muscle hardness (LMH) using a simple experimental design. The aim of the present study was to use this technique in order to determine LMH changes induced by a fatiguing prolonged contraction. A previous paper has ever modeled muscle mechanical properties assessed using elastography (Dresner et al., 2001). They started by recognizing that muscle has a given value of LMH when relaxed. The additional LMH due to contraction was then related to the number of linked cross-bridge and the total $\mathrm{LMH}$ was expressed as the sum of passive LMH and active LMH. Therefore, we managed to measure LMH in both active and passive conditions. Our results show that active LMH is not significantly changed during the fatiguing contraction, while the LMH at rest was significantly decreased immediately after the effort.

Concerning sEMG parameters, our results are in accordance with classical findings showing that muscle electrical activity increases while the MPF is decreased during a submaximal isometric contraction until exhaustion, indicating a muscle fatigue process (De Luca, 1984). While sEMG activity increased progressively during the fatiguing contraction, the mean RMS at the end of the test was less than the maximum recorded during maximal isometric contractions. Although it cannot be asserted using the present methodology, this result seems to indicate the presence of central fatigue at the end of the task failure (Behm and St-Pierre, 1997). On the other hand, the magnitudes of MPF decreases with fatigue were higher than $8 \%$ of initial values, which is classically accepted as the threshold for physiological relevance of changes in MPF (Oberg et al., 1990). Such decreases in MPF are related to peripheral fatigue (Masuda et al., 1999) that could be explained by a decrease in muscle fiber conduction velocity partially due to an accumulation of metabolites reducing intracellular pH (Brody et al., 1991; Lindstrom et al., 1977). In addition, the increase in FB1 could also be related to changes in recruitment strategies (Badier et al., 1993; Bendahan et al., 1996). Finally, it must be noted that changes in RMS, MPF and FB1were similar across the three considered muscles (Fig. 3), indicating that these superficial muscles were affected by central and peripheral fatigue. Considering the data in Fig. 3, it could be noted that the magnitudes of changes in sEMG parameters were lower for SO than for GM and GL, indicating a greater fatigability for both gastrocnemii. This result might be explained by the relatively higher percentage in slow fibers in SO (about $88 \%$ ) vs. GM (about 51\%) and GL (about 47\%) (Johnson et al., 1973; Kupa et al., 1995).

The RMS increase (Fig. 3a), and therefore the increase in the number of recruited fibers required to sustain a constant external torque, is not associated with changes in 
active LMH during the submaximal isometric effort (Fig. 4). Changes in LMH with fatigue are somewhat difficult to compare with previous studies of the literature since different experimental paradigms have been used, leading to different global stiffness measurements. For instance, Hunter and Kearney (1983) have shown that the ankle dynamic stiffness remains constant during prolonged constant isometric dorsiflexion. In contrast, Zhang and Rymer (2001) and Vigreux et al. (1980) have found a decrease in intrinsic joint stiffness and stiffness of series elastic component with fatigue. Zhang and Rymer (2001) considered that the reported decrease would reflect a reduction in stiffness of contractile elements. Interestingly, these authors also reported an increase in viscosity with fatigue that may be related to a decrease in muscle fiber relaxation rate. However, considering the global measurements performed by their study, several structures would be implied in these changes and LMH measurements performed in the present study do not support these hypotheses. Future experiments using elastography would enable researchers to assess the attenuation of the shear wave, which is related to the viscosity (Catheline et al., 2005).

Therefore, the LMH changes did not parallels sEMG changes during such a fatigue protocol, contrary to what was previously found for a non-fatiguing incremental isometric protocol (Gennisson et al., 2005). Assuming that muscle tension could remain quite constant during the submaximal fatiguing protocol, thanks to efficient motor units recruitment to counterbalance the fatigue of the first recruited motor units, it could be hypothesized that LMH would be further correlated to muscle tension (Jenkyn et al., 2003), rather than to muscle activity level. Nevertheless, this needs to be shown definitively. If so, transient elastography would be an efficient non-invasive tool to assess muscle specific tension of muscles. It would enable assess tension of both superficial and deep muscle of a muscle group, and could be useful in muscle modeling for direct simulations of muscle group contractions.

The ratio found between active LMH (at $40 \%$ of the MVIT) and LMH at rest $(2.1 \pm 0.6)$ is consistent with results of Gennisson et al. (2005), who have found a ratio of $3.08 \pm 2.20$ between biceps LMH at $50 \%$ of the maximal activity level and LMH at rest. Concerning LMH at rest, the averaged value found in the present study $(3.50 \mathrm{kPa})$ is in the same range but higher than that reported by Gennisson et al. (2005) at $0.92 \mathrm{kPa}$, and lower than that reported by Levinson et al. (1995) at $12 \mathrm{kPa}$. These relative discrepancies could be partially due to the different muscles tested in all of these studies (i.e. biceps brachii for Gennisson et al., quadriceps for Levinson et al., and gastrocnemius medialis in our study). Since sonoelastography was used by Levinson et al. (1995) while transient elastography was used in the two other studies, the different methods used could partially explain discrepancies in LMH measurements. The discrepancies between biceps brachii and gastrocnemius medialis LMH values, both obtained by transient elastography, might also be partially explained by the typological distributions of the considered muscles. Indeed, gastrocnemius medialis is usually composed of a higher percentage of type I fibers (Johnson et al., 1973), and the stiffness of fast fibers is known to be lower than stiffness of slow fibers (Goubel and Marini, 1987).

Immediately after the isometric contraction until exhaustion, LMH at rest was lower than before the test (Fig. 5). Two main hypotheses could be formulated to explain the lower value of LMH after the fatiguing protocol. (i) A possible increase in intra-muscular temperature induced by exercise could alter viscoelastic muscle-tendon properties (Kubo et al., 2001). Nevertheless, Magnusson et al. (2000) have shown that 30 min of continuous running induced a significant increase of $3.8^{\circ}$ in hamstrings intramuscular temperature, but did not affect viscoelastic hamstring properties. Consequently, the increase in intramuscular temperature induced by our fatiguing protocol should not have altered muscle viscoelastic properties. (ii) The elongation of tendon structures (Kubo et al., 2001; Maganaris et al., 2006), the increase in muscle pennation angle and the decrease in fascicle length (Mademli and Arampatzis, 2005; Maganaris et al., 2002) indicate that a creep phenomenon occurs in tendons during prolonged contractions. It has recently been shown that the creep does not induce changes in tendon and aponeurosis stiffness (Mademli et al., 2006). Nevertheless, our results concerning $\mathrm{LMH}$ at rest, which have shown that muscle elastic properties are also modified by a fatiguing contraction, could suggest that the creep would induce changes in intrinsic muscle mechanical properties. Further studies are necessary to examine this point and determine whether it could have an influence on muscle activity levels during the contraction.

Finally, our results show that LMH of the GM measured using transient elastography during a submaximal isometric plantar flexion until exhaustion is not significantly changed. It would show that the LMH would be further correlated to muscle tension rather than to muscle activity level indicating that LMH and future studies would be necessary to determine whether the LMH could be a non-invasive measurement of muscle tension considering different muscle types, muscle tasks and different populations. Finally, the LMH of the GM measured at rest was significantly decreased after the fatiguing protocol. This result could be explained by a creep phenomenon which has been reported in literature for the tendon during prolonged isometric contractions. Further investigations are required to determine whether it could have an influence on muscle activity levels during the contraction.

\section{Acknowledgements}

The authors thank Michel Roche for his technical assistance and Jonathan Douay for his help during experimentations. 


\section{References}

Badier M, Guillot C, Lagier-Tessonnier F, Burnet H, Jammes Y. EMG power spectrum of respiratory and skeletal muscles during static contraction in healthy man. Muscle and Nerve 1993;16:601-9.

Behm DG, St-Pierre DM. Effect of fatigue duration and muscle type on voluntary and evoked contractile properties. Journal of Applied Physiology 1997;82:1654-61.

Bendahan D, Jammes Y, Salvan AM, Badier M, Confort-Gouny S, Guillot C Cozzone PJ. Combined electromyography - 31P-magnetic resonance spectroscopy study of human muscle fatigue during static contraction. Muscle and Nerve 1996;19:715-21.

Brody LR, Pollock MT, Roy SH, De Luca CJ, Celli B. pH-induced effects on median frequency and conduction velocity of the myoelectric signal. Journal of Applied Physiology 1991;71:1878-85.

Catheline S, Gennisson JL, Delon G, Fink M, Sinkus R, Abouelkaram S, Culioli J. Measurement of viscoelastic properties of homogeneous soft solid using transient elastography: an inverse problem approach. Journal of the Acoustical Society of America 2005;116:3734-41.

De Luca CJ. Myoelectrical manifestations of localized muscular fatigue in humans. Critical Reviews in Biomedical Engineering 1984;11:251-79.

De Luca CJ. The use of surface electromyography in biomechanics. Journal of Applied Biomechanics 1997;13(2):135-63.

Dresner MA, Rose GH, Rossman PJ, Muthupillai R, Manduca A, Ehman RL. Magnetic resonance elastography of skeletal muscle. Journal of Magnetic Resonance Imaging 2001;13:269-76.

Fung YC. Biomechanics, Mechanical Properties of Living Tissues. 2nd ed. New York: Springer; 1993.

Fuglevand AJ, Zackowski KM, Huey KA, Enoka RM. Impairment of neuromuscular propagation during human fatiguing contractions at submaximal forces. Journal of Physiology 1993;460:549-72.

Gennisson JL, Cornu C, Catheline S, Fink M, Portero P. Human muscle hardness assessment during incremental isometric contraction using transient elastography. Journal of Biomechanics 2005;38:1543-50.

Goubel F, Marini JF. Fibre type transition and stiffness modification of soleus muscle of trained rats. Pflugers Archives 1987;410:321-5.

Heers G, Jenkyn T, Dresner MA, Klein MO, Basford JR, Kaufman KR, Ehman RL, An KN. Measurement of muscle activity with magnetic resonance elastography. Clinical Biomechanics 2003;18:537-42.

Hermens HJ, Freriks B, Disselhorst-Klug C, Rau G. Development of recommendations for SEMG sensors and sensor placement procedures. Journal of Electromyography and Kinesiology 2000;10:361-74.

Hewson DJ, Hogrel JY, Langeron Y, Duchêne J. Evolution in impedance at the electrode-skin interface of two types of surface electrodes during long-term recordings. Journal of Electromyography and Kinesiology 2003; 13:273-9.

Hof AL. Muscle mechanics and muscular control. Journal of Biomechanics 2003;36:1031-8.

Hunter IW, Kearney RE. Invariance of ankle dynamic stiffness during fatiguing muscle contractions. Journal of Biomechanics 1983;16:985-91.

Jenkyn TR, Ehman RL, An KN. Noninvasive muscle tension measurement using the novel technique of magnetic resonance elastography (MRE). Journal of Biomechanics 2003;36:1917-21.

Johnson MA, Polgar J, Weightman D, Appleton D. Data on the distribution of fibre types in thirty six human muscles. An autopsy study. Journal of the Neurological Sciences 1973;18(1):111-29.

Kubo K, Kanehisa H, Kawakami Y, Fukunaga T. Influences of repetitive muscle contractions with different modes on tendon elasticity in vivo. Journal of Applied Physiology 2001;91:277-82.

Kupa EJ, Roy SH, Kandarian SC, De Luca CJ. Effects of muscle fiber type and size on EMG median frequency and conduction velocity. Journal of Applied Physiology 1995;79:23-32.
Levinson SF, Shinagawa M, Sato T. Sonoelastic determination of human skeletal muscle elasticity. Journal of Biomechanics 1995;28:114554

Lindstrom L, Kadefors R, Petersen I. An electromyographic index for localized muscle fatigue. Journal of Applied Physiology 1977;43:750-4.

Mademli L, Arampatzis A. Behaviour of the human gastrocnemius muscle architecture during submaximal isometric fatigue. European Journal of Applied Physiology 2005;94:611-7.

Mademli L, Arampatzis A, Walsh M. Effect of muscle fatigue on the compliance of the gastrocnemius medialis tendon and aponeurosis. Journal of Biomechanics 2006;39:426-34.

Maganaris CN, Baltzopoulos V, Sargeant AJ. Repeated contractions alter the geometry of human skeletal muscle. Journal of Applied Physiology 2002;93:2089-94.

Maganaris CN, Baltzopoulos V, Sargeant AJ. Human calf muscle responses during repeated isometric plantarflexions. Journal of Biomechanics 2006;39:1249-55.

Magnusson SP, Aagaard P, Larsson B, Kjaer M. Passive energy absorption by human muscle-tendon unit is unaffected by increase in intramuscular temperature. Journal of Applied Physiology 2000;88:1215-20.

Maïsetti O, Guével A, Legros P, Hogrel JY. SEMG power spectrum changes during a sustained $50 \%$ maximum isometric torque do not depend upon the prior knowledge of the exercise duration. Journal of Electromyography and Kinesiology 2002a;12:103-9.

Maïsetti O, Guével A, Legros P, Hogrel JY. Prediction of endurance capacity of quadriceps muscles in humans using surface electromyogram spectrum analysis during submaximal voluntary isometric contractions. European Journal of Applied Physiology 2002b;87:509-19.

Masuda K, Masuda T, Sadomaya T, Inaki M, Katsuda S. Changes in surface EMG parameters during static and dynamic fatiguing contractions. Journal of Electromyography and Kinesiology 1999;9:39-46.

Oberg T, Sandsjö L, Kadefors R. Electromyogram mean power frequency in non-fatigued trapezius muscle. European Journal of Applied Physiology and Occupational Physiology 1990;61:362-9.

Sandrin L, Tanter M, Gennisson JL, Catheline S, Fink M. Shear elasticity probe for soft tissues using 1D transient elastography. IEEE Transactions in Ultrasonic, Ferroelectric and Frequency Control 2002;49:436-46.

Vigreux B, Cnockaert JC, Pertuzon E. Effects of fatigue on the series elastic component of human muscle. European Journal of Applied Physiology and Occupational Physiology 1980;45:11-7.

Wilson JW, Murphy AJ, Pryor FJ. Musculotendinous stiffness: its relationship to eccentric, isometric and concentric performance. Journal of Applied Physiology 1994;76:2714-9.

Zhang LQ, Rymer WZ. Reflex and intrinsic changes induced by fatigue of human elbow extensor muscles. Journal of Neurophysiology 2001;86:1086-94. 